\title{
Attenuation of septal lesion-induced shuttlebox facilitation by 5 -hydroxytryptophan
}

\author{
ROBERT F. SMITH \\ George Mason University, Fairfax, Virginia 22030
}

\begin{abstract}
Septal-lesioned rats and unoperated controls were tested for acquisition of a shuttlebox avoidance task. Each animal received either a $105-\mathrm{mg} / \mathrm{kg} 5$-hydroxytryptophan (5-HTP) or a saline injection prior to each day's testing. Septal animals with saline injections were significantly faster in learning the task. This facilitation was significantly attenuated by injections of 5-HTP. The data suggest that septal lesion-induced shuttlebox facilitation may be a function of the serotonin reduction previously reported to be a consequence of the lesion.
\end{abstract}

Á number of studies have shown that septal lesions enhance acquisition of a two-way shuttlebox avoidance task in rats (see Fried, 1972, for a review). Although this has been an established phenomenon for a number of years, efforts to determine the pharmacological basis for this septal lesion effect have not been successful. Kelsey (1975) concluded that the facilitation is not a result of alterations of pituitary-adrenal function which accompany septal lesions. Although scopolamine injection has been shown to cause similar enhancement on active avoidance (see Fried, 1972), identity of action of this cholinolytic drug with septal lesion has been inferred rather than directly demonstrated. A second neurochemical manipulation which causes enhancement of active avoidance acquisition is serotonin reduction. Such reduction by raphe lesion (Lorens, 1973), PCPA injection (Brody, 1970; Tenen, 1967), or lesions of the nucleus accumbens septi (Lorens, Sorenson, \& Harvey, 1970) results in enhanced shuttlebox avoidance. Since septal lesion also produces a reduction in forebrain serotonin content (Lints \& Harvey, 1969; Smith, 1979), it is plausible that such a serotonin reduction might be a critical factor in the enhancement of shuttlebox performance seen after septal lesion.

The present study investigated the hypothesis that the facilitation is dependent on serotonin reduction consequent to the lesion. Previous work (Smith, 1979) has shown that such reduction is reversed in the lesioned animals by doses of $105 \mathrm{mg} / \mathrm{kg}$ 5-hydroxytryptophan (5-HTP). Since 5-HTP is selectively active in increasing serotonin content, it is possible to determine whether the serotonin reduction which accompanies septal lesion is a critical determinant of

This research was supported by a Faculty Research Grant from George Mason University. I thank Ms. Judith McWilliams and Ms. Dawn Royall for testing the subjects, and Mr. Timothy Coffer for competent histological work. the enhanced shuttlebox acquisition. The present study was designed to determine if 5-HTP injection prior to training would attenuate the enhanced shuttlebox acqusition seen after septal lesion.

\section{METHOD}

\section{Subjects.}

The subjects were 32 naive male Sprague-Dawley rats (300$350 \mathrm{~g}$ ), housed singly and given ad-lib access to food and water. Eight animals were included in each of the four groups: septal lesion and saline injection; unoperate and saline injection; septal lesion and 5-HTP injection; and unoperate and 5-HTP injection.

\section{Surgical Procedures}

Under $45-\mathrm{mg} / \mathrm{kg}$ sodium pentobarbital anesthesia, the scalp was incised, burr holes drilled in the skull, and an insulated electrode (00 insect pin, Scotchkote insulation, $0.5-\mathrm{mm}$ exposed tip) stereotaxically positioned to $1.0 \mathrm{~mm}$ anterior to bregma, $.5 \mathrm{~mm}$ lateral to midline, and $5.0 \mathrm{~mm}$ below dorsal skull surface. Bilateral electrolytic lesions were effected by passage of $2.5 \mathrm{~mA}$ cathodal current for $30 \mathrm{sec}$. The subjects were allowed to recover 7-10 days before testing. Unoperated animals received no surgery or anesthesia.

\section{Injection Protocols}

The 5-HTP dosage of $105 \mathrm{mg} / \mathrm{kg}$ has been shown to restore forebrain serotonin content and footshock sensitivity to near normal (Smith, 1979). D, L-5-HTP (Regis Chemical Co.) was dissolved in physiological saline in a concentration of $10 \mathrm{mg} / \mathrm{ml}$ and injected in a dosage of $105 \mathrm{mg} / \mathrm{ml}$. Animals receiving saline were given an equal volume of saline. All injections were administered 30 min prior to testing on each day of testing.

\section{Apparatus}

The subjects were tested in a BRS/LVE RSC-044 shuttle cage. Scrambled footshock was provided to the cage floor by a Stoelting Model 521S shocker and scrambler. Electromechanical programming provided all timing and control functions.

\section{Procedure}

Each day's testing consisted of 30 trials separated by 30 -sec ITIs. On each trial, a $90-\mathrm{dB}$ Sonalert signaled the onset of the trial. If the subject crossed the barrier within $10 \mathrm{sec}$, the Sonalert was terminated and the trial automatically scored as an avoidance. If the rat failed to cross within $10 \mathrm{sec}$, a .15-mA scrambled foot- 
shock was delivered to the side of the cage that the animal was on. Responding after shock onset was scored as an escape, and automatically terminated the tone and shock. If the animal failed to escape within $60 \mathrm{sec}$ of shock onset, the trial was automatically terminated, and a new trial initiated after the 30 -sec ITI. Measures of latency were not taken. The subjects were tested daily for 25 days, or until a criterion of $27 / 30$ avoidances in a given day was reached.

\section{RESULTS}

\section{Histology}

All animals were sacrificed at the completion of testing, and perfused intracardially with $.9 \%$ saline followed by $10 \%$ Formalin in $.9 \%$ saline. Frozen serial sections were cut at $40 \mu \mathrm{m}$ through the extent of the lesion, and stained with cresyl violet/luxol blue. Examination of the stained sections indicated that all lesions were large and bilaterally symmetrical. Medial and lateral septal areas were substantially destroyed in all animals. Consistent ancillary damage included the posterior portion of the nucleus accumbens septi, columns of the fornix, and overlying corpus callosum. Some neocortical damage was observed in a few subjects, but there were no subjects sustaining either damage below the level of the anterior commissure or to the caudate nucleus. Lesion extent was assessed without reference to drug treatment and the extent of the lesion in animals given saline and 5-HTP injection was comparable.

\section{Behavior}

Following completion of testing, a trials-tosuccessive-criteria analysis was used for data analysis. For each animal, the number of days to reach the successively more difficult criteria of $70 \%, 80 \%$, and $90 \%$ avoidances on a given day was computed. Subjects not reaching a criterion within 25 days were scored as 26. Analysis of variance of the data revealed significant surgical $(\mathrm{F}=19.279, \mathrm{df}=1 / 28, \mathrm{p}<.01)$, drug $(F=9.625, \mathrm{df}=1 / 28, \mathrm{p}<.01)$, and Surgery by Drug interaction $(\mathrm{F}=5.353, \mathrm{df}=1 / 28, \mathrm{p}<.05)$ effects; the repeated measures effect was also significant $(F=12.126, d f=2 / 56, p<.01)$, but no interactions involving the repeated measure were significant (Fs $<1)$. As Figure 1 demonstrates, and NewmanKeuls post hoc tests confirmed, these patterns of differences were due to more rapid acquisition in the septal-saline animals, relative to all other groups. That is, septal lesion, not combined with 5-HTP injection, significantly enhanced acquisition relative to controls, while addition of 5-HTP to the septal preparation reversed the facilitation-septal animals were not statistically different from controls.

\section{DISCUSSION}

The results of the present study clearly demonstrate that injections of 5-HTP in septal-lesioned rats

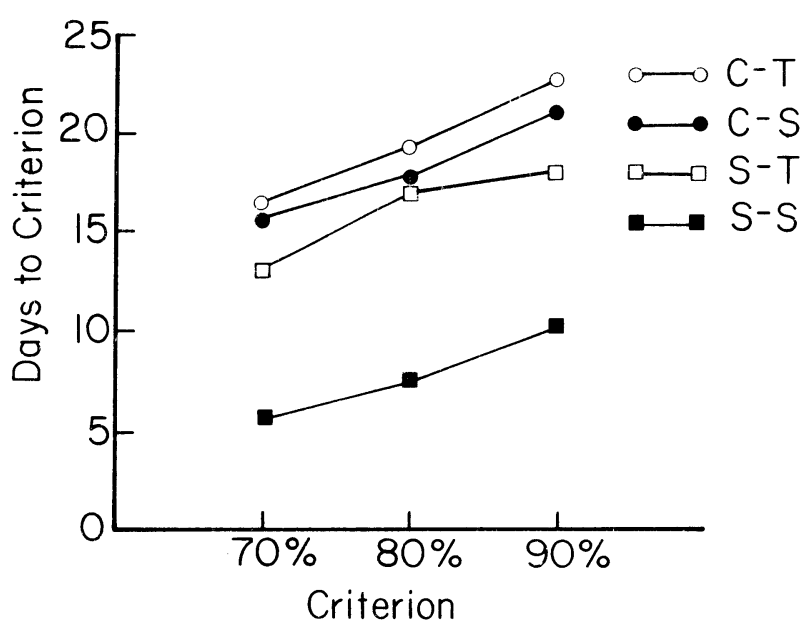

Figure 1. Days to successive criteria for each of the four treatment groups. Legend: $\mathrm{C}-\mathrm{T}=$ control, $5-\mathrm{HTP}$ injection; $\mathrm{C}-\mathrm{S}=$ control, saline injection; S-T = septal lesion and 5-HTP injection; S-S = septal lesion and saline injection.

significantly attenuate the lesion-induced facilitation on the shuttlebox avoidance task. Doses of 5-HTP used in the present study have been demonstrated to restore serotonin content and footshock sensitivity to near normal following septal lesion in animals of the same strain, sex, and age (Smith, 1979), but cause no overt changes in motoric behavior. The present findings suggest that enhanced avoidance learning following septal lesion may be due to reduced forebrain serotonin content, and consequent enhanced footshock sensitivity, which accompany septal lesion (Lints \& Harvey, 1969; Smith, 1979). A substantial literature confirms that serotonin reduction by a variety of manipulations produces enhanced footshock sensitivity (e.g., Lints \& Harvey, 1969; Lytle, Messing, Fisher, \& Phebus, 1976; Smith, 1979; Tenen, 1967). Several serotonin-reducing manipulations also enhance avoidance learning (e.g., Lorens, 1973; Tenen, 1967; the present data). The data of the present paper indicate that injections of 5-HTP, which have been previously found to restore footshock sensitivity and serotonin content to near normal in septal-lesioned animals, also attenuate the septal avoidance enhancement effect. Thus, the lesioninduced enhancement effect appears to depend on serotonin reduction consequent to lesion.

While the present data demonstrate the importance of serotonin reduction in the lesion-induced enhancement effect, they do not exclude the participation of other neurochemical alterations in the facilitation effect. In particular, a cholinergic system has been shown to also affect shuttlebox acquisition (see Fried, 1972), and recent demonstrations of interactions between the serotonergic and cholinergic systems in brain (Samanin, Quattrone, Peri, Ladinsky, \& Consolo, 1978) make the exclusion of other systems 
in the lesion-induced facilitation effect difficult to defend.

As previously noted (Smith, 1979), the involvement of the limbic projection of the serotonergic system in the modulation of sensitivity to footshock warrants caution in the interpretation of shock-motivated task data in animals with septal or hippocampal lesions. Although most such behavioral changes have been previously ascribed to reductions in the animal's ability to inhibit responding (see Douglas, 1975, for one review), it is equally plausible that enhanced footshock sensitivity consequent to serotonin reduction may in some cases be responsible for alterations in responding on shock-motivated tasks. The present study has demonstrated one such case where serotonin reduction consequent to the lesion appears to be responsible for lesion-induced alterations in motivated behavior.

\section{REFERENCES}

Brody, J. E., JR. Behavioral effects of serotonin depletion and of p-chlorophenylalanine (a serotonin depletor) in rats. Psychopharmacologia, 1970, 17, 14-33.

Douglas, R. J. The development of hippocampal function; implications for theory and for therapy. In R. L. Isaacson \& K. H. Pribram (Eds.), The hippocampus (Vol. 2). New York: Plenum, 1975.
Fried, P. A. Septum and behavior: A review. Psychological Bulletin, 1972, 78, 292-310.

KELSEY, J. E. Role of pituitary-adrenocortical system in mediating avoidance behavior of rats with septal lesions. Journal of Comparative \& Physiological Psychology, 1975, 88, 271-280.

Lints, C. E., \& Harvey, J. A. Altered sensitivity to electric shock and decreased brain content of serotonin following brain lesions in the rat. Journal of Comparative \& Physiological Psychology, 1969, 67, 23-31.

LorENS, S. A. Raphe lesions in cats: Forebrain serotonin avoidance behavior. Pharmacology, Biochemistry, \& Behavior, 1973, 1, 487-490.

Lorens, S. A., Sorenson, J. P., \& Harvey, J. A. Lesions in the nucleus accumbens septi: Behavioral and neurochemical effects. Journal of Comparative \& Physiological Psychology, 1970, 73, 284-290.

Lytle, L. D., Messing, R. B., Fisher, L., \& Phebus, L. Effects of long-term corn consumption on brain serotonin and the response to electric shock. Science, 1975, 190, 692-694.

Samanin, R., Quattrone, A., Peri, G., Ladinsky, H., \& Consolo, S. Evidence of an interaction between serotoninergic and cholinergic neurons in the corpus striatum and hippocampus of the rat brain. Brain Research, 1978, 151, 73-82.

Smith, R. F. Mediation of footshock sensitivity by serotonergic projection to hippocampus. Pharmacology, Biochemistry, \& Behavior, 1979, 10, 381-388.

TEnEN, S. S. The effects of p-chlorophenylalanine, a serotonin depletor, on avoidance acquisition, pain sensitivity, and related behavior in the rat. Psychopharmacologia, 1967, 10, 204-219.

(Received for publication March 13, 1979; revision accepted October $2,1979$. 